\title{
FINITELY ADDITIVE RADON-NIKODÝM THEOREM AND CONCENTRATION FUNCTION OF A PROBABILITY WITH RESPECT TO A PROBABILITY
}

\author{
PATRIZIA BERTI, EUGENIO REGAZZINI, AND PIETRO RIGO
}

(Communicated by William D. Sudderth)

\begin{abstract}
An "exact" Radon-Nikodým theorem is obtained for a pair $(m, \mu)$ of finitely-additive probabilities, using a notion of concentration function of $\mu$ with respect to $m$. In addition, some direct consequences of that theorem are examined.
\end{abstract}

\section{INTRODUCTION}

Throughout this paper the term probability will designate any positive, finitelyadditive measure on an algebra $\mathfrak{F}$ of subsets of $\Omega$, assuming value 1 at $\Omega$. We turn our attention to the case in which $\mu$ and $m$ are probabilities on $(\Omega, \mathfrak{F})$, with $\mu$ absolutely continuous with respect to (w.r.t.) $m$ [for each $\varepsilon>0$ there exists $\delta>0$ such that $\mu(E)<\varepsilon$ whenever $E \in \mathfrak{F}$ and $m(E)<\delta$ ]. Even if this condition holds, $\mu$ need not admit any "exact" Radon-Nikodým derivative w.r.t. $m$. Actually, under that hypothesis, Bochner [2] states that, given $\varepsilon>0$, there exists a simple function $f_{\varepsilon}$ such that $\left|\int_{E} f_{\varepsilon} d m-\mu(E)\right|<\varepsilon$ for all $E$ in $\mathfrak{F}$. Later, the same theorem is proved again by various authors at various times (cf. Bhaskara Rao and Bhaskara Rao [1, Chapter 6 and Notes and Comments to Chapter 6]). A few of them deal with the problem of finding necessary and sufficient conditions for the existence of exact Radon-Nikodým derivatives. In particular, Maynard [8] provides a complete solution to the problem at issue. De Finetti [5] already had pointed out the connections between the existence of an exact Radon-Nikodým derivative and certain properties of the lower boundary of the convex hull of the range of $(m, \mu)$, under the hypothesis that $\mathfrak{F}$ coincides with the power set of $\Omega$, and $m$ is strongly continuous [for each $\varepsilon>0$ there exists a partition $\left\{E_{1}, \ldots, E_{n}\right\}$ of $\Omega$ in $\mathfrak{F}$ such that $m\left(E_{i}\right)<\varepsilon$ for every $i]$. Recently, de Finetti's approach has been basically taken up again by

Received by the editors December 5, 1989.

1980 Mathematics Subject Classification (1985 Revision). Primary 60A10, 28A25, 28 A60.

Key words and phrases. Concentration function, extension, finitely-additive probability, RadonNikodým theorem.

The first author's research was partially supported by MPI (40\% 1985, Gruppo Nazionale "Processi Stocastici e Calcolo Stocastico").

The second author's research was partially supported by MPI (40\% 1987, Gruppo Nazionale "Modelli Probabilistici") and by CNR (Progetto Strategico "Statistica dei Processi Aleatori"). 
Candeloro and Martellotti [3], supposing that $m$ is strongly continuous, $\mathfrak{F}$ is a $\sigma$-algebra, and the exact derivative is picked within the class of real Borelmeasurable functions. In spite of these restrictions, essentially analogous to those originally considered by de Finetti, the above authors have resorted to some quite sophisticated instruments.

This paper aims at showing that de Finetti's [5] reasoning can be extended, with slight adjustments, to any pair $(m, \mu)$ of probabilities, not necessarily strongly continuous, on $(\Omega, \mathfrak{F})$. In particular, Candeloro and Martellotti's results follow from ours, which do rely solely on elementary facts.

Section 1 includes a few definitions that are used in subsequent sections; specifically, the concept of concentration function is expounded, because it characterizes de Finetti's approach. Section 2 exhibits some properties of the concentration function of $\mu$ w.r.t. $m$ when $\mu$ possesses an exact derivative w.r.t. $m$. Section 3 shows that these properties suffice for $\mu$ to admit a density w.r.t. $m$, or at least, w.r.t. some unique extension of $m$.

\section{Preliminaries}

The first result we quote is the extensibility of any probability from an algebra, of subsets of $\Omega$, to a larger one. Precisely, suppose $m$ is a probability on an algebra $\mathfrak{F}$ and $\mathfrak{F}^{*}$ an algebra of subsets of $\Omega$ with $\mathfrak{F}^{*} \supset \mathfrak{F}$; then there exists at least one probability $m^{*}$ on $\mathfrak{F}^{*}$, such that $m^{*}(A)=m(A)$ for all $A$ in $\mathfrak{F}$.

Many of our future developments are based on the notion of integrability in the sense of Dunford and Schwartz [6]. We recall this notion quoting from Bhaskara Rao and Bhaskara Rao [1, Chapter 4]. Let $m$ be a probability on $(\Omega, \mathfrak{F})$ and $f$ a simple function with representation $f=\sum_{i=1}^{k} c_{i} I_{E_{i}}$, for some real numbers $c_{1}, \ldots, c_{k}$ and partition $\left\{E_{1}, \ldots, E_{k}\right\}$ of $\Omega$ in $\mathfrak{F}$. Then, the real number $\sum_{i=1}^{k} c_{i} m\left(E_{i}\right)$ is said to be the integral of the simple function $f$. In order to define integrals of a larger class of functions, one can introduce the concept of hazy convergence of a sequence $\left\{f_{n}\right\}_{n=1}^{\infty}$ of functions from $\Omega$ to $\mathbf{R}$, according to which $f_{n} \rightarrow f$, as $n \rightarrow \infty$, if for each $\varepsilon>0$ :

$$
\inf \{m(B): B \in \mathfrak{F}, B \supset B(n, \varepsilon)\} \rightarrow 0 \text { as } n \rightarrow \infty \text {, }
$$

where $B(n, \varepsilon)=\left\{\omega \in \Omega:\left|f_{n}(\omega)-f(\omega)\right|>\varepsilon\right\}$. Then, a function $f: \Omega \rightarrow$ $[-\infty, \infty]$ is said to be integrable, in the sense of Dunford and Schwartz, if there exists a sequence $\left\{f_{n}\right\}_{n=1}^{\infty}$ of simple functions such that

(i) $\left\{f_{n}\right\}_{n=1}^{\infty}$ converges hazily to $f$ and

(ii) $\lim _{n, k \rightarrow \infty} \int_{\Omega}\left|f_{n}-f_{k}\right| d m=0$.

If $f$ is integrable, then the integral of $f$ is denoted by $\int_{\Omega} f d m$ and is defined to be the number $\lim _{n \rightarrow \infty} \int_{\Omega} f_{n} d m$; further, the sequence $\left\{f_{n}\right\}_{n=1}^{\infty}$ is called a determining sequence for $f$.

Another notion of great importance for forthcoming developments is that of concentration function. Given any pair of probabilities $(m, \mu)$ on $(\Omega, \mathfrak{F})$, let $R(m, \mu)$ denote its range in $\mathbf{R}^{2}, R_{*}(m, \mu)$ the convex hull of $R(m, \mu)$ and $\overline{R_{*}(m, \mu)}$ the closure of $R_{*}(m, \mu)$. The concentration function of $\mu$ w.r.t. $m$ is the function $\phi$ which associates every $x$ in $[0,1]$ with

$$
\phi(x)=\min \left\{y:(x, y) \in \overline{R_{*}(m, \mu)}\right\} .
$$

Clearly, $\phi$ is continuous, nondecreasing, and convex, with value 0 at 0 . Moreover, $\phi(1)=1$ if and only if $\mu$ is absolutely continuous w.r.t. $m$. 
We conclude this section by mentioning a certain subset of $[0,1]$ associated with $\phi$. Let $g$ be the left-hand derivative of $\phi, J$ the set of $x \in(0,1)$ such that $g$ has a jump at $x$, and $G$ the collection of $x \in(0,1)$ such that $g$ is strictly increasing at $x$ [i.e. $(g(y)-g(x)) /(y-x)>0$ whenever $y \neq x$ ]. The set:

$$
V=V(\phi)=J \cup G \cup\{1\}
$$

will play some role in the characterization of indefinite integrals w.r.t. probabilities.

\section{Properties of the Range of $(m, \mu)$ WHEN $\mu$ ADMITS AN EXACT DERIVATIVE WITH RESPECT TO $m$}

In this section we suppose that a nonnegative $m$-integrable function $f$ exists such that

$$
\int_{A} f(\omega) m(d \omega)=\mu(A) \text { for all } A \text { in } \mathfrak{F},
$$

and we expound a few simple properties of the range of $(m, \mu)$.

Let $\mathfrak{F}^{*}$ be any algebra, including $\mathfrak{F}$, w.r.t. which $f$ turns out to be Borelmeasurable. After selecting a probability $m^{*}$, which extends $m$ from $\mathfrak{F}$ to $\mathfrak{F}^{*}$, define the functions

$$
\begin{aligned}
& H(t)=m^{*}(\{\omega \in \Omega: f(\omega) \leq t\}) \quad t \in \mathbf{R}, \\
& q(x)=\inf \{t \in \mathbf{R}: H(t) \geq x\} \quad x \in(0,1) .
\end{aligned}
$$

Since $f$ is $m$-integrable, one can choose a sequence of simple functions, $\left\{f_{n}\right\}_{n=1}^{\infty}$, converging hazily to $f$ w.r.t. $m$ (cf. $\S 1$ ). Obviously, $\left\{f_{n}\right\}_{n=1}^{\infty}$ converges hazily to $f$ w.r.t. $m^{*}$ as well. Thus, at any continuity point $t$ of $H$, the latter can be expressed as

$$
H(t)=\lim _{n \rightarrow \infty} m\left(\left\{\omega \in \Omega: f_{n}(\omega) \leq t\right\}\right) .
$$

In view of (2.2), we are in a position to state

If $m_{1}^{*}$ and $m_{2}^{*}$ are extensions of $m$ from $\mathfrak{F}$ to $\mathfrak{F}^{*}$ and $(2.1)$ holds, then the set of continuity points of $H_{1}(t):=m_{1}^{*}(\{\omega \in$

(A) $\quad \Omega: f(\omega) \leq t\})$ and that of $H_{2}(t):=m_{2}^{*}(\{\omega \in \Omega: f(\omega) \leq t\})$ coincide, and $H_{1}=H_{2}$ on it. Consequently, the quantile function $q$ does not depend on the selected extension of $m$.

Because of (A), the function

$$
\psi(x):=\int_{0}^{x} q(y) d y \quad x \in[0,1]
$$

is, in its turn, independent of the selection of $m^{*}$. Plainly, $\psi$ is continuous, nondecreasing, and convex, with $\psi(0)=0$ and $\psi(1)=\int_{\Omega} f(\omega) m^{*}(d \omega)=1$. Our next goal lies in analysing the connections between $\psi$ and the concentration function of $\mu^{*}$ w.r.t. $m^{*}$, where $\mu^{*}$ denotes the extension of $\mu$ to $\mathfrak{F}^{*}$ given by

$$
\mu^{*}(E)=\int_{E} f(\omega) m^{*}(d \omega) \quad E \in \mathfrak{F}^{*}
$$


First, we point out that $(x, \psi(x)) \in R\left(m^{*}, \mu^{*}\right)$ whenever $x$ belongs to the range of $H$. In fact, suppose $x=H(t)$ for some $t$; then, setting $B_{x}=$ $\{\omega: f(\omega) \leq t\}$ yields $m^{*}\left(B_{x}\right)=x$ and

$$
\mu^{*}\left(B_{x}\right)=\int_{B_{x}} f d m^{*}=\int_{0}^{t} s d H(s)=\int_{0}^{t}[H(t)-H(s)] d s=\psi(x) .
$$

Second, $(x, \psi(x)) \in \overline{R\left(m^{*}, \mu^{*}\right)}$ in case $x=H(t-0)$ or $x=H(t+0)$ for some $t$. Indeed, take a sequence $\left\{t_{n}\right\}_{n=1}^{\infty}$ such that, respectively, $t_{n} \uparrow t$ or $t_{n} \downarrow t$, and define $B_{n}=\left\{\omega: f(\omega) \leq t_{n}\right\}$ and $x_{n}=H\left(t_{n}\right)$. Then $x_{n} \rightarrow x$, so that, by continuity of $\psi,\left(m^{*}\left(B_{n}\right), \mu^{*}\left(B_{n}\right)\right)=\left(x_{n}, \psi\left(x_{n}\right)\right) \rightarrow(x, \psi(x))$.

Third, for every $E \in \mathfrak{F}^{*}$ and $x \in(0,1), m^{*}(E) \geq x$ implies $\mu^{*}(E) \geq \psi(x)$. In fact, let $x \in[H(t-0), H(t+0)]$ for some $t$, and take any sequence $\left\{t_{n}\right\}_{n=1}^{\infty}$ such that $t_{n} \uparrow t$. Then, again setting $x_{n}=H\left(t_{n}\right)$ and $B_{n}=\left\{\omega: f(\omega) \leq t_{n}\right\}$, we obtain

$$
\begin{aligned}
\mu^{*}(E) & =\mu^{*}\left(B_{n}\right)+\int_{E \cap B_{n}^{c}} f d m^{*}-\int_{E^{c} \cap B_{n}} f d m^{*} \\
& \geq \mu^{*}\left(B_{n}\right)+t_{n} m^{*}\left(E \cap B_{n}^{c}\right)-t_{n} m^{*}\left(E^{c} \cap B_{n}\right) \\
& =\psi\left(x_{n}\right)+t_{n}\left\{m^{*}(E)-m^{*}\left(B_{n}\right)\right\} \\
& \geq \psi\left(x_{n}\right)+t_{n}\left(x-x_{n}\right) \rightarrow \psi(x) \text { as } n \rightarrow \infty .
\end{aligned}
$$

Let $\phi^{*}$ be the concentration function of $\mu^{*}$ w.r.t. $m^{*}$. The third of the above remarks implies that $\psi(x) \leq \phi^{*}(x)$ for all $x$, while the first and the second assure that $\psi(x) \geq \phi^{*}(x)$ whenever $x$ is adherent to the range of $H$. In particular, $\psi(x)=\phi^{*}(x)$ for every $x$ not interior to an interval on which $\psi$ is linear. Consequently, since $\psi \leq \phi^{*}$, and since $\psi$ and $\phi^{*}$ are both convex, it must be $\psi(x)=\phi^{*}(x)$ for each $x$ in $[0,1]$.

Now let us turn to the concentration function, $\phi$, of $\mu$ w.r.t. $m$. If $x=$ $H(t-0)$ or $x=H(t+0)$ for some $t$, it is always possible to select the above sequence $\left\{t_{n}\right\}_{n=1}^{\infty}$ in the set of continuity points of $H$. In that case, using relation (2.2), for each $\varepsilon>0$ a set $B \in \mathfrak{F}$ can be found such that $|m(B)-x|<$ $\varepsilon$ and $|\mu(B)-\psi(x)|<\varepsilon$. Consequently $(x, \psi(x)) \in \overline{R(m, \mu)}$, and since $\phi \geq \phi^{*}=\psi$, this proves that $\phi=\psi$. In short,

Suppose (2.1) holds and let $m^{*}$ be an extension of $m$ from

$\mathfrak{F}$ to $\mathfrak{F}^{*}$; then, assigning $\mu^{*}$ according to (2.3) implies that $\phi=\phi^{*}=\psi$, where $\phi\left(\phi^{*}\right)$ is the concentration function of $\mu$ $\left(\mu^{*}\right)$ w.r.t. $m\left(m^{*}\right)$.

Let us consider the set $V$ defined in (1.1). In view of (B), $V=V(\psi)$. On the other hand, thanks to the left-continuity of the quantile function $q$, one deduces that $q$ coincides with the left-hand derivative of $\psi$. Thus, $V$ can be equivalently referred to $q$. For our purposes, another point concerning $V$ is stressed.

$$
V \subset\{H(t): H \text { is continuous at } t\} \cup\{1\} .
$$

Proof. Let $L=\{H(t): H$ is continuous at $t\}$ and $x \in(0,1)$. If $q(x)<$ $q(x+0)$, then $x=H(t)$ for each $t \in(q(x), q(x+0))$, and $x$ belongs to $L$. Now suppose that $x$ is not in $L$. The previous argument implies that $q$ does not have a jump at $x$. On the other hand, there exists $t$ such that $H$ has a 
jump at $t$ and $x \in[H(t-0), H(t+0)]$, which gives $q(x)=q(y)$ for some $y \neq x$. Hence $x \notin V$, so that $V \subset L \cup\{1\}$.

By virtue of (C), if $x \in V \cap(0,1)$ then $x=H(t)$ for some continuity point $t$ of $H$, and consequently, setting $B_{x}=\{\omega: f(\omega) \leq t\}, m^{*}\left(B_{x}\right)=x$ whatever the extension $m^{*}$ of $m$ is.

To summarize this section, we have shown that if $(m, \mu)$ satisfies $(2.1)$ on $(\Omega, \mathfrak{F})$, then an algebra $\mathfrak{F}^{*} \supset \mathfrak{F}$ and a collection $\left\{B_{x}: x \in V\right\} \subset \mathfrak{F}^{*}$ are available, where $B_{1}=\Omega$ and $V=V(\phi)$, which satisfy the following condition: for every probability $m^{*}$, extending $m$ to $\mathfrak{F}^{*}$, there is a probability $\mu^{*}$, extending $\mu$ to $\mathfrak{F}^{*}$, such that

$$
\phi^{*}=\phi, \text { and } m^{*}\left(B_{x}\right)=x, \mu^{*}\left(B_{x}\right)=\phi^{*}(x) \text { for every } x \text { in } V .
$$

It should also be stressed that under the condition just quoted, $m$ has a unique extension to the algebra, $\mathfrak{F}_{0}$, generated by $\mathfrak{F}$ and $\left\{B_{x}: x \in V\right\}$; this fact, however, is proved in the next section (cf. Theorem 3.1).

\section{EXACT RADON-NIKODÝM THEOREM FOR PROBABILITIES}

This section aims at obtaining an exact Radon-Nikodým theorem for probabilities and at analysing some of its consequences. The idea that is used in proving our main results draws on $\S \S 6,7$ of de Finetti [5].

In what follows $(m, \mu)$ are probabilities on $(\Omega, \mathfrak{F}), \phi$ is the concentration function of $\mu$ w.r.t. $m$, and $g$ is the left-hand derivative of $\phi$. We will essentially be concerned with the next condition (here formulated for a generic pair of probabilities $(\nu, \lambda)$, defined on any algebra $\mathscr{A}$ of subsets of $\Omega, \phi$ denoting the concentration function of $\lambda$ w.r.t. $\nu)$ :

$(\alpha)$ there exists a collection $\left\{B_{x}: x \in V\right\} \subset \mathscr{A}$, where $B_{1}=\Omega$ and $V=$ $V(\phi)$, such that

$$
\nu\left(B_{x}\right)=x, \lambda\left(B_{x}\right)=\phi(x) \text { for every } x \text { in } V .
$$

Let us start by noting that, up to null sets, $\left\{B_{x}: x \in V\right\}$ is a nested collection of sets.

Lemma 3.1. If $(m, \mu)$ fulfills condition $(\alpha)$, then $m\left(B_{x} \backslash B_{y}\right)=0$ whenever $x, y \in V$ and $x \leq y$.

Proof. Let $x, y \in V, x \leq y$, and $a:=m\left(B_{x} \backslash B_{y}\right)$. Being $y+a=m\left(B_{y}\right)+a=$ $m\left(B_{x} \cup B_{y}\right)$ and $x-a=m\left(B_{x}\right)-a=m\left(B_{x} \cap B_{y}\right)$, the definition of concentration function implies that

$$
\begin{aligned}
\phi(y+a)-\phi(y) & \leq \mu\left(B_{x} \cup B_{y}\right)-\mu\left(B_{y}\right) \\
& =\mu\left(B_{x}\right)-\mu\left(B_{x} \cap B_{y}\right) \leq \phi(x)-\phi(x-a) .
\end{aligned}
$$

Since $\phi$ is convex, the inequality in (3.1) can always be reverted; consequently, $\phi(y+a)-\phi(y)=\phi(x)-\phi(x-a)$. If $a>0$, such equality implies that $\phi$ is linear on the interval $(x-a, y+a)$, and this gives $x, y \notin V$. Hence, $m\left(B_{x} \backslash B_{y}\right)=a=0$.

If condition $(\alpha)$ holds for $(m, \mu)$, then $\phi(1)=\mu\left(B_{1}\right)=\mu(\Omega)=1$. Thus, under condition $(\alpha), \mu$ is bound to be absolutely continuous w.r.t. $m$; actually, as we will show, $(\alpha)$ implies that $\mu$ possesses an exact derivative w.r.t. $m$. 
Lemma 3.2. Suppose condition $(\alpha)$ holds for $(m, \mu)$. Then,

$$
\mu(E)=\int_{E} f(\omega) m(d \omega)
$$

for all $E$ in $\mathfrak{F}$, where: $f(\omega)=g\left(x_{\omega}\right)$ and $x_{\omega}$ is defined by relation (3.2).

Proof. Set $g(0):=\inf \{g(x): x>0\}, A=\left\{t: \exists x_{1} \neq x_{2}\right.$ with $g\left(x_{1}\right)=t=$ $\left.g\left(x_{2}\right)\right\}$, and note that card $A \leq \operatorname{card} \mathbf{N}$. For every $n \in \mathbf{N}$, pick a number $M_{n} \in(n-1, n]$ with $g(0)+M_{n} \notin A$. Also, choose a subdivision of the interval $\left[g(0), g(0)+M_{n}\right]$, composed of $N=N(n)=n 3^{n}$ subintervals whose extreme points, $g(0):=t(0, n)<t(1, n)<\cdots<t(N, n):=g(0)+M_{n}$, are such that

(i) $t(1, n), t(2, n), \ldots, t(N-1, n)$ are not in $A$;

(ii) $t(i, n)=t(3 i, n+1)$ for $i=0,1, \ldots, N$;

(iii) $\max \{t(i, n)-t(i-1, n): i=1, \ldots, N\} \leq 2^{-n}$.

Define $x(i, n)=\sup \{x \in[0,1]: g(x) \leq t(i, n)\}, n \in \mathbf{N}$ and $i=1, \ldots, N$. The points $x(i, n)$ are in $V$ for all $n$ and $i$. In fact, if $x(i, n)=1$ then $x(i, n) \in V$. Otherwise, since $g$ is left-continuous, it must be either $g(x(i, n))$ $<t(i, n)$ or $g(x(i, n))=t(i, n)$. In the latter case, since $t(i, n) \notin A$ then $x(i, n)$ belongs to $V$; in the former, $g$ has a jump at $x(i, n)$ so that $x(i, n)$ is again in $V$.

After setting $Z_{n}:=\{x(i, n): i=1, \ldots, N(n)\} \cup\{1\}$ and $Z:=\bigcup_{n>1} Z_{n}$, we go on to define a subclass of $\mathfrak{F}:\left\{C_{x}: x \in Z\right\}$, such that $C_{1}=\Omega, m\left(C_{x}\right)=$ $x, \mu\left(C_{x}\right)=\phi(x)$, and $C_{x} \subset C_{y}$ for every $x, y$ in $Z$ with $x \leq y$. This definition is based on the following procedure that we describe with reference to the interval $[a, b] \subset[0,1]$ and to the points $y_{1} \leq \cdots \leq y_{r}$ belonging to $[a, b] \cap V$. After putting $y_{0}=a$ and $y_{r+1}=b$, the procedure introduces the sets

$$
F_{x}:=\left\{B_{x} \cap\left(\bigcap_{y_{j} \geq x} B_{y_{j}}\right)\right\} \cup\left(\bigcup_{y_{j} \leq x} B_{y_{j}}\right) \quad x \in[a, b] \cap V .
$$

In view of Lemma 3.1: $m\left(F_{x}\right)=x, \mu\left(F_{x}\right)=\phi(x)$ and $F_{x} \subset F_{y}$ whenever $x \in\left[y_{i-1}, y_{i}\right]$ and $y \in\left[y_{j}, y_{j+1}\right]$ for $i=1, \ldots, r$ and $j=i, \ldots, r$.

Now, assuming $C_{1}=\Omega$, let us apply the previous procedure to $[0,1]$ and to $y_{i}=x(i, 1), i=1,2,3$, and let us set $C_{x(i, 1)}=F_{x(i, 1)}^{(1)}$ for $i=1,2,3$, where $F_{x}^{(1)}$ denotes the output of the procedure associated with $x \in V$. Now let us apply the same procedure to each one of the intervals $[0, x(1,1)]$, $[x(1,1), x(2,1)],[x(2,1), x(3,1)],[x(3,1), 1]$ and to the points $y_{i}=$ $x(i, 2), i=1, \ldots, 18$, with the proviso that the sets $B_{x}$ are replaced by the $F_{x}^{(1)}$ 's. After denoting the output of this application of the procedure by $F_{x}^{(2)}$, one obtains $F_{x(3 i, 2)}^{(2)}=F_{x(i, 1)}^{(1)}, i=1,2,3$, so that, after putting $C_{x(i, 2)}:=F_{x(i, 2)}^{(2)},\left\{C_{x}: x \in Z_{2}\right\} \supset\left\{C_{x}: x \in Z_{1}\right\}$.

Proceeding in this manner, after $n-1$ steps we obtain $\left\{F_{x}^{(n-1)}: x \in V\right\}$ with $C_{x(i, n-1)}:=F_{x(i, n-1)}^{(n-1)}, i=1, \ldots, N(n-1)$, and are able to apply the previous procedure to each one of the intervals $[0, x(1, n-1)],[x(1, n-1)$, $x(2, n-1)], \ldots,[x(N(n-1), n-1), 1]$ and to the points $y_{i}=x(i, n), i=1$, $\ldots, N(n)$, with $F_{x}^{(n-1)}$ in the place of $B_{x}$ for each $x$ in $V$. Since $F_{x(i, n-1)}^{(n-1)}=$ 
$F_{x(3 i, n)}^{(n)}$, we set: $C_{x(i, n)}=F_{x(i, n)}^{(n)}$ for $i=1, \ldots, N(n)$, so that $\left\{C_{x}: x \in Z_{n}\right\} \supset$ $\left\{C_{x}: x \in Z_{n-1}\right\}$.

At this point, let us put, for every $n \in \mathbf{N}$,

$$
E_{1, n}=C_{x(1, n)}, E_{i, n}=C_{x(i, n)} \backslash C_{x(i-1, n)} \text { for } i=2, \ldots, N(n)
$$

and

$$
f_{n}(\omega)=\sum_{i=1}^{N(n)} t(i-1, n) I_{E_{i, n}}(\omega) \quad \omega \in \Omega .
$$

By construction, $\left\{f_{n}\right\}_{n=1}^{\infty}$ is an increasing sequence of simple functions converging to $f$ hazily w.r.t. $m$, where

$$
f(\omega)=g\left(x_{\omega}\right), \quad x_{\omega}=\inf \left\{x \in Z: \omega \in C_{x}\right\} \quad \omega \in \Omega .
$$

Indeed, if $\omega$ is in $E_{1, n}$, then $x_{\omega} \leq x(1, n)$, so that $g(0) \leq g\left(x_{\omega}\right) \leq$ $g(x(1, n)) \leq g(0)+2^{-n}$. Otherwise, if $\omega$ is in $E_{i, n}, i=2, \ldots, N$, then $x_{\omega} \in$ $[x(i-1, n), x(i, n)]$. On the other hand, since $t(i-1, n)<g\left(x_{\omega}\right) \leq t(i, n)$ whenever $x(i-1, n)<x_{\omega} \leq x(i, n)$, and since $t(i, n)-t(i-1, n) \leq 2^{-n}$, one deduces

$$
\left\{\omega:\left|f(\omega)-f_{n}(\omega)\right|>2^{-n}\right\} \subset C_{x(N, n)}^{c} \cup\left(\bigcup_{i=2}^{N(n)}\left\{\omega \in E_{i, n}: x_{\omega}=x(i-1, n)\right\}\right) .
$$

Let $\gamma(A):=\inf \{m(B): A \subset B, B \in \mathfrak{F}\}$ be the outer measure of $A \subset \Omega$ associated with $m$. If $\omega \in E_{i, n}$ and $x_{\omega}=x(i-1, n), i=2, \ldots, N(n)$, then $\omega$ must belong to $C_{x} \backslash C_{x(i-1, n)}$ for every $x \in Z$ with $x>x(i-1, n)$. Moreover, since $\omega \notin C_{x(i-1, n)}$, the definition of $x_{\omega}$ implies that there exist points of $Z$ arbitrarily close to, but strictly greater than, $x(i-1, n)$. Hence

$$
\begin{aligned}
\gamma\left(\left\{\omega \in E_{i, n}: x_{\omega}=x(i-1, n)\right\}\right) & \leq x-x(i-1, n) \\
& \text { for each } x>x(i-1, n), i=2, \ldots, N(n)
\end{aligned}
$$

which gives

$$
\gamma\left(\left\{\omega:\left|f(\omega)-f_{n}(\omega)\right|>2^{-n}\right\}\right) \leq 1-x(N(n), n) \rightarrow 0, \quad \text { as } n \rightarrow \infty .
$$

We will show that $\int_{E} f_{n} d m \rightarrow \mu(E)$, for every $E$ in $\mathfrak{F}$, as $n \rightarrow \infty$. Fix $n \in \mathbf{N}, i=1, \ldots, N(n)$, and set $x(0, n)=0, C_{0}=\varnothing$; the convexity of $\phi$ implies: $\phi(x(i-1, n)) \geq \phi(x(i, n))+g(x(i, n))(x(i-1, n)-x(i, n))$, so that

$$
\begin{aligned}
\mu\left(E_{i, n}\right) & =\phi(x(i, n))-\phi(x(i-1, n)) \\
& \leq g(x(i, n))(x(i, n)-x(i-1, n)) \leq t(i, n) m\left(E_{i, n}\right) .
\end{aligned}
$$

Let $E \in \mathfrak{F}$ and $a:=m\left(C_{x(i-1, n)} \cup\left(E \cap E_{i, n}\right)\right)$. Then

$$
\phi(a) \leq \mu\left(C_{x(i-1, n)} \cup\left(E \cap E_{i, n}\right)\right)=\phi(x(i-1, n))+\mu\left(E \cap E_{i, n}\right)
$$

which, denoting the right-hand derivative of $\phi$ by $\phi_{+}^{\prime}$ and assuming that $x(i-1, n)<x(i, n)$, yields

$$
\begin{aligned}
t(i-1, n) m\left(E \cap E_{i, n}\right) & \leq \phi_{+}^{\prime}(x(i-1, n))(a-x(i-1, n)) \\
& \leq \phi(a)-\phi(x(i-1, n)) \leq \mu\left(E \cap E_{i, n}\right) .
\end{aligned}
$$

Further, if $x(i-1, n)=x(i, n)$, then $t(i-1, n) m\left(E \cap E_{i, n}\right)=0=\mu\left(E \cap E_{i, n}\right)$, and (3.4) still holds. From (3.3) and (3.4) it follows that: $\mu\left(E \cap E_{i, n}\right)=$ 
$\mu\left(E_{i, n}\right)-\mu\left(E^{c} \cap E_{i, n}\right) \leq t(i, n) m\left(E_{i, n}\right)-t(i-1, n) m\left(E^{c} \cap E_{i, n}\right) \leq 2^{-n} m\left(E_{i, n}\right)+$ $t(i-1, n) m\left(E \cap E_{i, n}\right)$. Together with (3.4) the last inequality gives

$$
0 \leq \mu\left(E \cap E_{i, n}\right)-t(i-1, n) m\left(E \cap E_{i, n}\right) \leq 2^{-n} m\left(E_{i, n}\right) .
$$

Noting that $0 \leq \mu\left(E \cap C_{x(N, n)}^{c}\right) \leq \mu\left(C_{x(N, n)}^{c}\right)=1-\phi(x(N, n))$, and summing (3.5) for $i=1, \ldots, N(n)$, one obtains

$$
0 \leq \mu(E)-\int_{E} f_{n} d m \leq 2^{-n} x(N(n), n)+1-\phi(x(N(n), n)) .
$$

By the continuity of $\phi, x(N(n), n) \rightarrow 1$ implies that $\phi(x(N(n), n)) \rightarrow \phi(1)=$ 1 ; hence $\int_{E} f_{n} d m \rightarrow \mu(E)$.

Since $\left\{f_{n}\right\}_{n=1}^{\infty}$ is a monotonic sequence, and since $\int_{\Omega} f_{n} d m \rightarrow \mu(\Omega)$, one easily verifies that $\int_{\Omega}\left|f_{n}-f_{k}\right| d m \rightarrow 0$ as $n, k \rightarrow \infty$. Consequently, $f$ is $m$-integrable and $\left\{f_{n}\right\}_{n=1}^{\infty}$ is a determining sequence for $f$ (cf. $\S 1$ ), so that: $\int_{E} f d m=\lim _{n \rightarrow \infty} \int_{E} f_{n} d m=\mu(E)$ for all $E$ in $\mathfrak{F}$, and this completes the proof.

Our next goal is to show that the necessary condition, pointed out at the end of $\S 2$, is sufficient for $\mu$ to have a density w.r.t. $m$. More precisely, we will find that this statement holds provided that we introduce a slight modification of the usual notion of density of $\mu$ w.r.t. $m$.

In order to describe such a modification, let us note that the above-mentioned condition states the existence of an algebra $\mathfrak{F}_{0} \supset \mathfrak{F}$, such that $m$ admits a unique extension, $m_{0}$, from $\mathfrak{F}$ to $\mathfrak{F}_{0}$. Moreover, it states the existence of an $m_{0}$-integrable function $f$ such that $\int_{E} f d m_{0}=\mu(E)$ for every $E$ in $\mathfrak{F}$. Because of the uniqueness of the extension of $m$ to $\mathfrak{F}_{0}$, it seems reasonable to view the possible lack of a density of $\mu$ w.r.t. $m$ as immaterial. Hence, in such a case, we say that $\mu$ has a density w.r.t. $m$ on $\mathfrak{F}_{0}$ and write $\int_{E} f d m$ in the place of $\int_{E} f d m_{0}$, for every $E$ in $\mathfrak{F}$. We adopt this notion of density to state the following exact Radon-Nikodým theorem for finitely-additive probabilities.

Theorem 3.1. Suppose that an algebra $\mathfrak{F}^{*} \supset \mathfrak{F}$ and a collection $\left\{B_{x}: x \in V\right\} \subset$ $\mathfrak{F}^{*}$ are available, where $B_{1}=\Omega$ and $V=V(\phi)$, satisfying the following condition: for every probability $m^{*}$, extending $m$ to $\mathfrak{F}^{*}$, there is a probability $\mu^{*}$, extending $\mu$ to $\mathfrak{F}^{*}$, such that $\phi^{*}=\phi$ and condition $(\alpha)$ holds for $\left(m^{*}, \mu^{*}\right)$ ( $\phi^{*}$ being the concentration function of $\mu^{*}$ w.r.t. $m^{*}$ ). Then $m$ has a unique extension to the algebra $\mathfrak{F}_{0}$, generated by $\mathfrak{F}$ and $\left\{B_{x}: x \in V\right\}$, and $\mu$ has $a$ density w.r.t. $m$ on $\mathfrak{F}_{0}$.

Proof. By Lemma 3.2, $\mu(E)=\int_{E} f d m^{*}$ for all $E$ in $\mathfrak{F}$, whatever the extension $m^{*}$ of $m$ is. Also, thanks to its construction, $f$ is integrable w.r.t. the restriction of $m^{*}$ to $\mathfrak{F}_{0}$ (cf. proof of Lemma 3.2). Finally, the uniqueness of the extension of $m$ to $\mathfrak{F}_{0}$ is an immediate consequence of the following fact.

Claim. If $x \in(0,1)$ is not interior to any interval of linearity for $\phi$ and $B \in \mathfrak{F}^{*}$, then all the probabilities $\left(m^{*}, \mu^{*}\right)$ on $\mathfrak{F}^{*}$, extending $(m, \mu)$ and such that $\phi^{*}=\phi, m^{*}(B)=x, \mu^{*}(B)=\phi^{*}(x)$, coincide on the algebra generated by $\mathfrak{F}$ and $B$.

Proof of the claim. Let $\left\{r_{n}\right\}_{n=1}^{\infty}$ and $\left\{s_{n}\right\}_{n=1}^{\infty}$ be two sequences in $[0,1]$ such that $r_{n} \uparrow x$ and $s_{n} \downarrow x$. For each $n$, define $A_{n}=\left\{(t, y): t=a r_{n}+\right.$ $\left.(1-a) s_{n}, a \in[0,1], \phi(t) \leq y<a \phi\left(r_{n}\right)+(1-a) \phi\left(s_{n}\right)\right\}$. Since $x$ is not 
interior to any interval of linearity of $\phi, A_{n}$ is nonempty; moreover, $A_{n} \subset$ $\overline{R_{*}(m, \mu)}$ and $\overline{R_{*}(m, \mu)} \backslash A_{n}$ is convex and closed, so that there is $D_{n} \in \mathfrak{F}$ with $\left(m\left(D_{n}\right), \mu\left(D_{n}\right)\right) \in A_{n}$. Trivially, $m\left(D_{n}\right) \rightarrow x$ and $\mu\left(D_{n}\right) \rightarrow \phi(x)$. Now it is sufficient to show that, if an extension $\left(m^{*}, \mu^{*}\right)$ of $(m, \mu)$ is such that $\phi^{*}=\phi$, $m^{*}(B)=x$ and $\mu^{*}(B)=\phi^{*}(x)$, then $m^{*}(E \cap B)=\lim _{n \rightarrow \infty} m\left(E \cap D_{n}\right)$ for each $E$ in $\mathfrak{F}$. As it is easily verifiable, it is enough to show that $\lim _{n \rightarrow \infty} m^{*}\left(D_{n} \Delta B\right)=$ 0 . Hence, we are proving that $\lim _{n \rightarrow \infty} m^{*}\left(D_{n} \backslash B\right)=0$; in a similar way, it can be shown that $\lim _{n \rightarrow \infty} m^{*}\left(B \backslash D_{n}\right)=0$. Let $h_{n}=m^{*}\left(D_{n} \backslash B\right)$; then $m^{*}\left(D_{n} \cup B\right)=x+h_{n}$ and $m^{*}\left(D_{n} \cap B\right)=m\left(D_{n}\right)-h_{n}$, so that

$$
\begin{aligned}
\mu\left(D_{n}\right)-\phi\left(m\left(D_{n}\right)-h_{n}\right) & \geq \mu\left(D_{n}\right)-\mu^{*}\left(D_{n} \cap B\right) \\
& =\mu^{*}\left(D_{n} \cup B\right)-\phi(x) \geq \phi\left(x+h_{n}\right)-\phi(x) .
\end{aligned}
$$

Let $\left\{h_{n_{j}}\right\}$ be a subsequence of $\left\{h_{n}\right\}$ converging to $h:=\limsup _{n \rightarrow \infty} h_{n} \geq 0$; applying (3.6) to the sets $D_{n_{j}}$ and taking the limit as $j \rightarrow \infty$, one deduces $\phi(x)-\phi(x-h) \geq \phi(x+h)-\phi(x)$, and, in view of the convexity of $\phi: \phi(x)-$ $\phi(x-h)=\phi(x+h)-\phi(x)$. But $x$ is not interior to any interval of linearity for $\phi$ and consequently $0=h=\lim _{n \rightarrow \infty} m^{*}\left(D_{n} \backslash B\right)$.

Finally, we analyse two implications of Lemma 3.2.

First, we state a generalization of Theorem 3.3 of Candeloro and Martellotti [3]. Unlike the original version, the following corollary does not assume the strong continuity of $m$.

Corollary 3.1. Let $\mathfrak{F}$ be a $\sigma$-algebra of subsets of $\Omega$ and $(m, \mu)$ probabilities on $(\Omega, \mathfrak{F})$. There exists a Borel-measurable and m-integrable function $f$ on $\Omega$ such that $\mu(E)=\int_{E} f d m$ for each $E \in \mathfrak{F}$, if, and only if, condition $(\alpha)$ holds for $(m, \mu)$.

Proof. The "only if" part follows by repeating the argument of $\S 2$ with $\mathfrak{F}^{*}=\mathfrak{F}$. Conversely, under condition $(\alpha)$, Lemma 3.2 implies that $\omega \rightarrow f(\omega)=g\left(x_{\omega}\right)$ is an exact derivative of $\mu$ w.r.t. $m$. Further, being $\mathfrak{F}$ a $\sigma$-algebra, $\left\{\omega: x_{\omega}<\right.$ $a\}$ is in $\mathfrak{F}$ for every real $a$. Therefore, using $g$ is monotone, $f$ is Borelmeasurable.

Second, we deduce the classical Radon-Nikodým theorem. To do this, we note that, if $\mathfrak{F}$ is a $\sigma$-algebra and $(m, \mu)$ are $\sigma$-additive probabilities on $(\Omega, \mathfrak{F})$, then $R(m, \mu)$ is a closed subset of $\mathbf{R}^{2}$ [7]. But, the closedness of $R(m, \mu)$ and the absolute continuity of $\mu$ w.r.t. $m$ are sufficient for the existence of a density, as it is proved by the following corollary of Lemma 3.2. By the way, in the case of probabilities such a corollary represents a slight generalization of Corollary 3.2 of Candeloro and Martellotti [4].

Corollary 3.2. Let $(m, \mu)$ be probabilities on $(\Omega, \mathfrak{F})$ with $\mu$ absolutely continuous w.r.t. $m$. If $R_{*}(m, \mu) \supset \overline{R(m, \mu)}$, which is trivially true when $R(m, \mu)$ is closed, then there exists an m-integrable function $f$ on $\Omega$ such that $\mu(E)=$ $\int_{E} f d m$ for each $E$ in $\mathfrak{F}$. Moreover, if $\mathfrak{F}$ is a $\sigma$-algebra, then $f$ can be taken Borel-measurable.

Proof. A point $P$, belonging to any subset $S$ of $\mathbf{R}^{2}$, is said to be extremal for $S$ if $P$ cannot be obtained as a nontrivial convex combination of two points in $S$. Let $A=R(m, \mu)$. By Lemma 3.2 and Corollary 3.1, it is sufficient to prove that $\phi(1)=1$ and $(x, \phi(x)) \in A$ whenever $x$ is in $V \cap(0,1)$. To 
this purpose note that, if $0 \leq y<x<1$, then $(x, y) \in \partial\left(\overline{A_{*}}\right)$ if and only if $y=\phi(x)$. Further, if $P \in \partial\left(\overline{A_{*}}\right)$ and $P=a P_{1}+(1-a) P_{2}$ for some $a \in(0,1)$ and $P_{1}, P_{2} \in \overline{A_{*}}$, then $\partial\left(\overline{A_{*}}\right)$ contains the whole segment connecting $P_{1}$ with $P_{2}$. It follows that if $x \in(0,1)$ and $(x, \phi(x))$ is not extremal for $\overline{A_{*}}$, then $x$ is interior to an interval of linearity for $\phi$. Let $x \in V \cap(0,1)$. The previous argument implies that $(x, \phi(x))$ is an extremal point for $\overline{A_{*}}$. But $\overline{A_{*}}=(\bar{A})_{*}$ (since $A$ is bounded), and $(\bar{A})_{*}=A_{*}$ (since $\left.A_{*} \supset \bar{A}\right)$. Thus $(x, \phi(x))$ is extremal for $A_{*}$, which implies $(x, \phi(x)) \in A$. Finally, being $\mu$ absolutely continuous w.r.t. $m, \phi(1)=1$.

\section{REFERENCES}

1. K. P. S. Bhaskara Rao and M. Bhaskara Rao, Theory of charges, Academic Press, London, 1983.

2. S. Bochner, Additive set functions on groups, Ann. Math. 40 (1939), 769-799.

3. D. Candeloro and A. Martellotti, A Radon-Nikodým theorem for finitely additive measures, Adv. in Math. (to appear).

4. __ A Radon-Nikodým theorem for vector-valued finitely additive measures with closed range, Technical Report, Dip. Matematica, Università di Perugia, 1988.

5. B. de Finetti, La struttura delle distribuzioni in un insieme astratto qualsiasi, Giorn. Ist. Ital. Attuari 18 (1955), 15-28; English transl., Probability, induction and statistics, J. Wiley, 1972.

6. N. Dunford and J. T. Schwartz, Linear operators, Part I: General theory, Wiley, New York, 1958.

7. A. A. Liapounoff, Sur les fonctions-vecteurs complètement additives, Izv. Akad. Nauk. SSSR 4 (1940), 465-478. (Russian)

8. H. B. Maynard, A Radon-Nikodým theorem for finitely additive bounded measures, Pacific J. Math. 83 (1979), 401-413.

Dipartimento Statistico, via Curtatone, 1, 50123 Firenze, Italy

Consiglio Nazionale delle Ricerche-Istituto PER le Applicazioni della Matematica E DELL' INFormatica, viA AMPÈRE, 56, 20131 Milano, ItAly 